\title{
Anlotinib can overcome acquired resistance to EGFR-TKIs via FGFR1 signalling in non-small cell lung cancer without harboring EGFR T790M mutation
}

\section{Zengzhi Lian}

the First Affiliated Hospital of Soochow University

\section{Wenwen Du}

First Affiliated Hospital of Soochow University

\section{Yang Zhang}

First Affiliated Hospital of Soochow University

\section{Yulong Fu}

First Affiliated Hospital of Soochow University

Ting Liu

First Affiliated Hospital of Soochow University

\section{Anqi Wang}

First Affiliated Hospital of Soochow University

\section{Tingting Cai}

First Affiliated Hospital of Soochow University

\section{Jianjie Zhu}

First Affiliated Hospital of Soochow University

\section{Yuanyuan Zeng}

First Affiliated Hospital of Soochow University

\section{Zeyi Liu ( $\nabla$ liuzeyisuda@163.com )}

First Affiliated Hospital of Soochow University https://orcid.org/0000-0003-2528-6909

Jian-an Huang

First Affiliated Hospital of Soochow University

\section{Research}

Keywords: anlotinib, EGFR-TKI, resistance, FGFR1, non-small cell lung cancer

Posted Date: February 11th, 2020

DOI: https://doi.org/10.21203/rs.2.23141/v1 
License: (c) (i) This work is licensed under a Creative Commons Attribution 4.0 International License. Read Full License 


\section{Abstract}

Background Although many studies have defined mechanisms of resistance to EGFR-TKIs, acquired resistance remains the major limitation of monotherapy with EGFR-TKIs. Therefore, there is an urgent need to develop effective therapeutic interventions to overcome acquired resistance.

Methods Cell viability was analysed using CCK-8 assay. EGFR T790M mutation was sequenced on a HiSeq 4000 platform. mRNA from HCC827 and HCC827 gefitinib-resistant (GR) cells was analysed by genome analyser-based deep sequencing. The effect of anlotinib on apoptosis and cell cycle arrest of HCC827 GR was detected by fluorescence-activated cell sorting (FACS) analysis. A mouse xenograft model was used to assess the killing effect of anlotinib on HCC827 GR cells.

Results Here we found the T790M mutation in the PC-9 gefitinib-resistant (GR) cell line but not in the HCC827 GR cell line. Interestingly, anlotinib could suppress the growth of HCC827 GR cells by inhibiting FGFR1 in vitro and in a mouse xenograft model. Moreover, FGFR1 was overexpressed in HCC827 GR cells, and the knockdown of FGFR1 reversed gefitinib resistance in HCC827 GR cells. Furthermore, anlotinib induced apoptosis and cell cycle arrest in HCC827 GR cells by increasing the activity of Caspase- 3 and decreasing the expression of Cyclin D1.

Conclusion FGFR1 overexpression is one of the mechanisms of EGFR-TKI acquired resistance and anlotinib can suppresses the growth of EGFR-TKI-resistant NSCLC cells without T790M mutation.

\section{Introduction}

Lung cancer is the major cause of cancer-related death throughout the world. ${ }^{1}$ Non-small cell lung cancer (NSCLC) accounts for $85 \%$ of lung cancers. The five-year survival rate for NSCLC is less than $15 \%{ }^{2}$ Epidermal growth factor receptor (EGFR)-sensitizing mutations have been used for the selection of patients with advanced NSCLC for EGFR tyrosine kinase inhibitor (EGFR-TKI) treatment. ${ }^{3}$ Numerous clinical studies have shown that EGFR-TKIs exhibit a better clinical benefit than traditional therapeutic strategies-chemotherapy and radiotherapy. ${ }^{4}$ Nevertheless, all responding patients invariably acquire resistance following the initial response within 8-12 months of therapy. ${ }^{5}$ Several acquire resistance mechanisms that include secondary mutation in EGFR (T790M), ${ }^{6}$ KRAS mutation, ${ }^{7}$ PIK3CA mutation, ${ }^{8}$ MET amplification, ${ }^{9}$ epithelial-mesenchymal transition (EMT) and small cell lung cancer (SCLC) transformation have been revealed. ${ }^{10}$ However, the possibility that other mechanisms are involved in resistance to EGFR-TKls cannot be excluded, and the need to develop effective therapeutic interventions to overcome acquired resistance is urgent.

The fibroblast growth factor receptor family (FGFR1-4) belongs to the RTK family, and there are 18 different FGF ligands. ${ }^{11,12}$ FGFR is involved in many physiological processes, including wound repair, embryogenesis and angiogenesis. ${ }^{13}$ Dysregulation of FGFR1 to tumorigenesis, transformation, and tumour progression has been reported in a broad range of malignancies. ${ }^{14,15}$ The activation of FGFR1 
can promote EMT in prostate cancer, breast cancer and FGFR1-amplified lung cancer. ${ }^{16-18}$ FGF/FGFR signalling pathway alterations have been connected with chemotherapy resistance and poor clinical outcome. ${ }^{19,20}$

Anlotinib is a multi-target receptor TKI in various cancers that has a broad spectrum of inhibitory actions against tumour growth and angiogenesis. ${ }^{21,22}$ Numerous studies have indicated that anlotinib suppresses tumour growth via the inhibition of c-Kit, Ret, Aurora-B, c-FMS and DDR1. Furthermore, anlotinib inhibits the angiogenesis of tumour cells by selectively targeting VEGFR (1, 2 and 3), PDGFR (a and $\beta$ ) and FGFR (1, 2, 3 and 4). ${ }^{23-26}$ Clinical trials have demonstrated that anlotinib therapy prolongs progression-free survival (PFS) and overall survival (OS) in refractory advanced NSCLC patients subjected to third-line or beyond third-line therapy. One study reported that anlotinib therapy was beneficial for prolonging OS in NSCLC patients harbouring EGFR mutations, especially patients harbouring the EFGR T790M mutation. Moreover, anlotinib inhibited angiogenesis in an H1975-derived xenograft model via inhibiting CCL2. ${ }^{21}$ However, the effect of anlotinib on NSCLC patients with EGFR-TKI resistance due to non-T790M mutation and the mechanism of this effect remain unclear.

In this study, we demonstrate that anlotinib inhibited the growth of EGFR-TKI-resistant NSCLC cells without T790M mutation in vitro and in a mouse xenograft model. Furthermore, anlotinib induced apoptosis and cycle arrest in EGFR-TKI-resistant NSCLC cells by inhibiting FGFR1. These results provide novel alternatives and therapeutic strategies for patients with EGFR-TKI acquired resistance.

\section{Materials And Methods}

\section{Cell culture and reagents}

HCC827 cells were purchased from the Cell Bank of the Chinese Academy of Sciences (Shanghai, China). To establish gefitinib-resistant (GR) HCC827 (HCC827 GR) cell strain, HCC827 cells were exposed to gefitinib according to the methods of Koizumi et al. ${ }^{27}$ PC-9 and GR PC9 (PC9 GR) cell lines were obtained from Professor Caicun Zhou as a gift. HCC827 and HCC827 GR cell lines were routinely cultured in RPMI1640 medium supplemented with 10\% foetal bovine serum (Gibco, Carlsbad, CA, USA). The PC-9 and PC9 GR cell lines were cultured in DMEM containing $10 \%$ foetal bovine serum. All cells were cultured in a humidified incubator containing $5 \% \mathrm{CO}_{2}$ at $37^{\circ} \mathrm{C}$. Gefitinib was purchased from Selleck (Selleck Chemicals, Houston, TX, USA). Anlotinib was given as a gift from Chia Tai Tianqing Pharmaceutical Group Co., Ltd. (Jiangsu, China).

\section{Next-generation DNA sequencing}

We profiled DNA from cells by using a capture-based targeted sequencing panel. Human genomic regions 1.4 megabases in total size including selected exons and introns of 416 genes were captured using 120base pair (bp) probes. DNA was fragmented into segments 200 to $250 \mathrm{bp}$ in length, captured by the 120bp probes, and sequenced by obtaining paired $2 \times 150$-bp reads. After DNA extraction using the QIAamp 
DNA FFPE Tissue Kit (Qiagen, Hilden, Germany), DNA concentrations were measured by using the Qubit dsDNA assay (Invitrogen, Carlsbad, CA). The DNA quality was confirmed by ensuring that the A260/A280 ratio was 1.8:2.0. DNA was hybridized with the capture probes (the bait), selected using magnetic beads, and polymerase chain reaction (PCR) amplified. Then, a bioanalyser (Qubit and Agilent 2100, Agilent Technologies, Santa Clara, CA) was used to perform high-sensitivity assays assessing DNA quality and size range. All samples were sequenced on a HiSeq 4000 platform (Illumina, Inc., San Diego, CA); we obtained pair-end reads.

\section{Cell viability assay}

Cells $\left(3 \times 10^{3}\right.$ cells/well) were seeded in 96-well plates overnight. Anlotinib or gefitinib was added at different doses, and the cells were incubated for $72 \mathrm{~h}$. Then, a Cell Counting Kit-8 assay kit (CCK-8, Boster, Wuhan, China) was used according to the manufacturer's instructions to assess cell viability. Each sample was plated in triplicate, three independent experiments were performed, and IC50 was defined as the concentration needed for a $50 \%$ reduction in the absorbance.

\section{Quantitative real-time PCR (qRT-PCR)}

Total RNA was reverse transcribed using reverse transcriptase M-MLV (TaKaRa, Shiga, Japan) according to the manufacturer's protocol. qRT-PCR was performed using SYBR Premix ExTaq ${ }^{\text {TM }}$ (Takara). The primer sequences for qRT-PCR of FGFR1 and GAPDH were as follows: AXL, sense: 5'-

TAATGGACTCTGTGGTGCCCTC-3', anti-sense: 5'-ATGTGTGGTTGATGCTGCCG-3'; GAPDH, sense: 5'TGCACCACCAACTGCTTAGC-3', anti-sense: 5'-GGCATGGACTGTG GTCATGG-3'. The PCR programme used was as follows: $50^{\circ} \mathrm{C}$ for $2 \mathrm{~min}$ and $95^{\circ} \mathrm{C}$ for $10 \mathrm{~min}$ followed by 45 cycles of $95^{\circ} \mathrm{C}$ for $15 \mathrm{sec}$ and $60^{\circ} \mathrm{C}$ for $1 \mathrm{~min}$. FGFR1 mRNA expression values were normalized to those of the internal control GAPDH. Relative expression was calculated using the cycle threshold (Ct) method.

\section{Western blot analysis}

Cells were lysed on ice with RIPA lysis buffer containing protease and phosphatase inhibitor for 20 min. The lysates were collected by high-speed centrifugation at $12000 \mathrm{rpm}$ for $15 \mathrm{~min}$ at $4{ }^{\circ} \mathrm{C}$. The protein concentration was measured using a BCA Protein Assay kit (Thermo Scientific, Rockford, IL, USA). The extracts were mixed with SDS sample buffer and subjected to $10 \%$ SDS-PAGE. Following electrophoretic transfer onto nitrocellulose, the membrane was blocked with 5\% skim milk in TBS for 1 hour and then incubated with primary antibodies at $4{ }^{\circ} \mathrm{C}$ overnight. The membranes were washed thoroughly with Trisbuffered saline with Tween-20 (TBST) and then incubated with peroxidase-conjugated secondary antibody for 2 hours at room temperature. All antibodies except anti-FRFR1 and anti-pFRFR1 were purchased from Cell Signaling Technology (Danvers, MA, USA). Anti-FRFR1 and anti-pFRFR1 were purchased from Merck (Billerica, MA, USA).

\section{Flow cytometry analysis}

HCC827 GR cells were seeded in 6-well plates. Then, the cells were treated with anlotinib at different concentrations. As a control, cells were treated with the vehicle, DMSO. We analysed cell apoptosis and 
the cell cycle status of the cells by using Annexin V-FITC and propidium iodide (PI) (fluorescein isothiocyanate, R\&D Systems) staining according to the manufacturer's protocol.

\section{Cell transfection}

Transfection was carried out using Lipofectamine 2000 transfection reagent (Invitrogen, Waltham, MA, USA) according to the manufacturer's protocols. All siRNAs were purchased from GenePharma Co. (Shanghai, China). The target siRNA sequences were as follows: si-NC, sense: 5'-

UUCUCCGAACGUGUCACGU-3', anti-sense: 5'-ACGUGACACGUUCGGAGAA-3'; si-FGFR1, sense: 5'CGGUCAUCGUCUACAAGAU-3', anti-sense: 5'-AUCUUGUAGACGAUGACCG-3'.

\section{Xenograft mouse model}

$\mathrm{BALB} / \mathrm{C}$ athymic nude mice (female, 3-4 weeks old and 16-20 $\mathrm{g}$ in weight) were purchased from the Experimental Animal Center of Soochow University and bred under pathogen-free conditions. HCC827 GR cells $\left(2.5 \times 10^{6}\right)$ were subcutaneously injected into the fore axillary region. Tumour volume was calculated using the following formula: volume = length $\times$ width $^{2} / 2$. When tumour volumes reached approximately $200 \mathrm{~mm}^{3}$, the mice were randomly allocated into groups of six animals and administered anlotinib ( $2 \mathrm{mg} / \mathrm{kg}$ ), gefitinib ( $4 \mathrm{mg} / \mathrm{kg}$ ) or vehicle by oral gavage every two days for 12 days. Tumour volume was measured once a day. All animals were handled in strict accordance with good animal practice as defined by the Guide for the Care and Use of Experimental Animals from the Experimental Animal Center of Soochow University, and all protocols were approved (2017.165) by the Animal Ethical and Welfare Committee of the First Affiliated Hospital of Soochow University.

\section{Statistical analysis}

All obtained results are presented as the mean \pm standard deviation (SD). Statistical significance was analysed with Student's t-test, and $\mathrm{P}<0.05$ indicated significance. All statistical analyses were performed using SPSS 7.0 software (SPSS, Chicago, IL, USA) and GraphPad Prism 7.0 (GraphPad, San Diego, CA, USA).

\section{Results}

\section{Gefitinib resistance in HCC827 GR cells is not due to EGFR T790M mutation}

Our previous study found an activating deletion in exon 19 in the HCC827 and PC-9 cell lines. ${ }^{28}$ The T790M mutation in EGFR is a known mechanism of acquired gefitinib resistance in lung cancer. ${ }^{6}$ To determine whether there the GR strains had the T790M mutation, we first examined the sensitivity of HCC827 and PC-9 parental and GR cells to gefitinib using a CCK-8 assay. The viability of HCC827 GR and PC-9 GR cells was unaffected by increasing concentrations of gefitinib up to $10 \mu \mathrm{M}($ IC50 $>10 \mu \mathrm{M})$, whereas HCC827 and PC-9 cells were sensitive to gefitinib (Fig. 1A and 1B). We then analysed EGFR for 
DNA substitution corresponding to the T790M mutation. As shown in Fig. 1C, the T790M mutation was found in the PC-9 GR cell line, while we found no evidence for this mutation in the HCC827 GR cell line (Fig. 1C and Supplemental Table 1). These results suggest that the T790M mutation is responsible for resistance to gefitinib in PC9 GR cells rather than HCC827 GR cells, and other mechanisms contribute to resistance to gefitinib in HCC827 GR cells.

\section{FGFR1 expression is higher in HCC827 GR cells than in the parental HCC827 cells}

To explore the gefitinib resistance mechanism in HCC827 GR cells, mRNA from HCC827 and HCC827 GR cells was submitted for RNA sequencing (RNA-seq) analysis by genome analyser-based deep sequencing. The resulting analysis showed that the mRNA expression level of FGFR1 was higher in HCC827 GR cells than in parental HCC827 cells (Fig. 2A). Western blot and qRT-PCR analyses confirmed that FGFR1 expression was increased in HCC827 GR cells (Fig. 2B). In contrast, FGFR1 expression was lower in PC-9 GR cells than in parental PC-9 cells (Supplemental Fig. 1). We next examined the inhibitory effect of anlotinib on the growth of HCC827 GR cells. As shown in Fig. 2C, the inhibitory effect of anlotinib on HCC827 GR cells was stronger than that on parental HCC827 cells. To verify that anlotinib inhibited the growth of HCC827 GR cells through targeting FGFR1, we detected phosphorylated FGFR1 and FGFR1 in HCC827 GR cells treated with anlotinib at different concentrations by Western blot analysis. As shown in Fig. 2D and 2E, anlotinib inhibited the phosphorylation of FGFR1 in HCC827 GR cells, and this inhibitory effect was stronger than that on parental HCC827 cells. In addition, anlotinib inhibited the phosphorylation of AKT and ERK in both HCC827 and HCC827 GR cells.

\section{Anlotinib induces apoptosis and cell cycle arrest in HCC827 GR cells}

We conducted fluorescence-activated cell sorting (FACS) analysis to determine whether anlotinib can induce apoptosis and cell cycle arrest in HCC827 GR cells. HCC827 GR cells were treated with anlotinib at gradually increasing concentrations, As shown in Fig. 3A and 3B, when the dose of anlotinib reached $1 \mu \mathrm{M}$, the proportion of HCC827 GR cells at $\mathrm{G}_{2} / \mathrm{M}$ phase and apoptosis increased significantly. When the dose reached $5 \mu \mathrm{M}$, the proportion of $\mathrm{HCC} 827 \mathrm{GR}$ cells at $\mathrm{G}_{0} / \mathrm{G}_{1}$ phase decreased significantly. Western blot analysis indicated that anlotinib induced a marked increase in the activity of Caspase-3 and decrease in the expression of Cyclin D1 in HCC827 GR cells (Fig. 3C).

\section{Knockdown of FGFR1 reverses gefitinib resistance in HCC827 GR cells}

We evaluated the effect of FGFR1 on gefitinib resistance in HCC827 GR cells by the siRNA-mediated knockdown of FGFR1. First, we succeeded in knocking down FGFR1 with siRNA in HCC827 GR cells (Fig. 4A). The growth rate of the FGFR1 interference group was slower than that of the control group 
(Fig. 4B). The CCK-8 assay indicated that HCC827 GR cells transfected with si-FGFR1 were more sensitive to gefitinib and more resistant to anlotinib than the control group (Fig. 4C and 4D). Transfection of siFGFR1 in HCC827 GR cells enhanced the gefitinib-induced downregulation of $p$-EGFR and p-AKT but not the downregulation of p-ERK (Fig. 4E and 4F).

\section{Anlotinib inhibits the growth of HCC827 GR cells in vivo}

To investigate whether anlotinib inhibits the growth of HCC827 GR cells in vivo, we inoculated athymic nude mice with HCC827 GR cells and then administered $2 \mathrm{mg} / \mathrm{kg}$ anlotinib, $4 \mathrm{mg} / \mathrm{kg}$ gefitinib or vehicle control. As shown in Fig. 5A, the administration of anlotinib had the strongest inhibitory effect on tumour growth, and the tumours began to shrink on the third day, whereas the tumours in the gefitinibadministered group began to grow on the fifth day. In addition, compared to those in the gefitinibadministered group, the tumours in the anlotinib-administered group were significantly smaller and lighter after treatment for 12 days (Fig. $5 \mathrm{~B}$ and $5 \mathrm{C}$ ). Resected tissues from the treated xenograft tumours were analysed by Western blotting to confirm that anlotinib inhibited the phosphorylation of FGFR1, AKT and ERK (Fig. 5D).

\section{Discussion}

Almost all NSCLC patients treated with EGFR-TKI inevitably develop acquired resistance. ${ }^{29,} 30$ Although many studies have defined mechanisms of resistance to EGFR-TKIs, including secondary mutations in EGFR (T790M), ${ }^{6}$ KRAS mutations, ${ }^{7}$ PIK3CA mutations, ${ }^{8}$ MET amplification, ${ }^{9}$ EMT and SCLC transformation, ${ }^{10}$ acquired resistance remains the major limitation of monotherapy with EGFR-TKI. Therefore, there is an urgent need to develop effective therapeutic interventions to overcome acquired resistance. Osimertinib monotherapy is the currently recommended second-line treatment for EGFR T790M mutation-positive NSCLC. However, there is no drug to treat NSCLC with other mechanisms leading to acquired resistance. In this study, we found that anlotinib inhibited the growth of EGFR-TKIresistant NSCLC cells without harbouring T790M mutation in vitro and in a mouse xenograft model. Furthermore, anlotinib induced apoptosis and cycle arrest in EGFR-TKI-resistant NSCLC cells by inhibiting FGFR1. These results provide novel alternatives and therapeutic strategies for non-T790M-mutated, EGFR-TKI-resistant NSCLC patients.

Anlotinib is an oral multi-target receptor TKI. In vitro studies using recombinant enzymes indicated that anlotinib selectively inhibits VEGFRs, which are crucial proteins in both vasculogenesis and angiogenesis. Anlotinib suppresses tumour cell proliferation via the inhibition of PDGFR ( $a$ and $\beta$ ), c-Kit, and Ret as well as DDR1, Aurora-B and c-FMS, a group of kinase targets involved in tumour progression. ${ }^{23-26}$ One study reported that anlotinib therapy was beneficial for prolonging OS in NSCLC patients harbouring EGFR mutations, especially patients harbouring the EGFR T790M mutation. Moreover, anlotinib inhibited angiogenesis in an H1975-derived xenograft model via inhibiting CCL2. ${ }^{21}$ However, the effect of anlotinib 
on NSCLC patients with EGFR-TKI resistance due to non-T790M mutation and the mechanism of this effect remain unclear.

To examine the inhibitory effect of anlotinib on EGFR-TKI-resistant NSCLC cells without T790M mutation, we exposed HCC827 and PC-9 cells to gefitinib to establish the GR cell lines HCC827 GR and PC-9 GR. Then, we analysed EGFR for the DNA substitution corresponding to the T790M mutation. The T790M mutation was found in the PC-9 GR cell line but not in the HCC827 GR cell line. In addition, anlotinib inhibited the growth of HCC827 GR cells in vitro and in a mouse xenograft model. Furthermore, anlotinib induced apoptosis and cycle arrest in HCC827 GR cells by increasing the activity of Caspase-3 and decreasing the expression of Cyclin D1. These results suggested that anlotinib suppresses the growth of EGFR-TKI-resistant NSCLC without T790M mutation.

The FGFR family belongs to the RTK family, and there are 18 different FGF ligands. ${ }^{11,}{ }^{12}$ FGFR is involved in many physiological processes, including wound repair, embryogenesis and angiogenesis. ${ }^{13}$ Activation of the FGF/FGFR signalling pathway promotes cancer progression and enhances the angiogenic potential of the tumour microenvironment. ${ }^{31-33}$ Dysregulation of FGFR1 leading to tumorigenesis, transformation, and tumour progression has been reported in a broad range of malignancies. ${ }^{14,15}$ The activation of FGFR1 can promote EMT in prostate cancer, breast cancer and FGFR1-amplified lung cancer. ${ }^{16-18}$ FGF/FGFR signalling alterations have been connected with chemotherapy resistance and poor clinical outcome. ${ }^{19}, 20$ We found that FGFR1 was overexpressed in HCC827 GR cells and that knockdown of FGFR1 reversed gefitinib resistance in HCC827 GR cells. Moreover, anlotinib inhibited FGFR1 in HCC827 GR cells in vitro and in a mouse xenograft model. These results suggested that the overexpression of FGFR1 is one of the mechanisms of EGFR-TKI acquired resistance and that anlotinib inhibits the FGFR1 signalling pathway in HCC827 GR cells.

There are some limitations to our study. First, the anti-tumour effect of anlotinib was confirmed in only EGFR-TKI-resistant HCC827 cells. However, our findings should be validated in various EGFR-TKI-resistant lung cancers. Second, because anlotinib is a multi-target receptor TKI, whether anlotinib inhibits the growth of EGFR-TKI-resistant NSCLC cells through other targets should be further investigated in future studies. Third, we determined that the molecular mechanism by which anlotinib inhibits the growth of EGFR-TKI-resistant NSCLC cells in animal and cellular models. To expand the application of anlotinib as a preclinical cancer drug, we will need to further verify the effect of anlotinib in patient-derived xenograft (PDX) models of EGFR-TKI-resistant NSCLC.

In summary, we demonstrate that the overexpression of FGFR1 is one of the mechanisms of EGFR-TKI acquired resistance and that anlotinib suppresses the growth of EGFR-TKI-resistant NSCLC cells without T790M mutation in vitro and in a mouse xenograft model by inhibiting FGFR1. Our study suggests anlotinib as a rational therapeutic drug for non-T790M-mutated, EGFR-TKI-resistant NSCLC patients.

\section{Declarations}




\section{Ethics approval and consent to participate}

All animal experiments were carried out in accordance with the Guide for the Care and Use of Experimental Animals from the Experimental Animal Center of Soochow University.

\section{Consent for publication}

We obtained consent to publish from the participant.

\section{Availability of data and materials}

The authors will make readily reproducible materials described in the manuscript, including software, databases and all relevant raw data are freely available to scientists.

\section{Competing interests}

The authors declare that they have no competing interests.

\section{Funding}

This work was supported by grants from the Science and Technology Plan Project of Suzhou (No. SYS201749), the Suzhou Key Laboratory for Respiratory Medicine (No. SZS201617), the Clinical Medical Center of Suzhou (No. Szzx201502), the Jiangsu Provincial Key Medical Discipline (No. ZDXKB2016007), the National Natural Science Foundation of China (No. 81802885, No. 81702870), and the Clinical Key Specialty Project of China.

\section{Authors' Contributions}

ZZL and YLF were involved in the acquisition and analysis of data. WWD and YZ participated in the writing of the manuscript and the interpretation of data. JJZ, YYZ, TL, AQW and TTC were involved in the interpretation and analysis of data, ZYL and JAH designed the study and finalized the manuscript. All authors read and approved the final manuscript.

\section{Acknowledgements}




\section{Disclosure}

The abstract of this paper was presented at the World Conference on Lung Cancer as a poster presentation with interim findings. The poster's abstract was published in "Poster Abstracts" in Annals of Oncology. DOI: https://doi.org/10.1093/annonc/mdz063.027.

\section{References}

[1] Bray F, Ferlay J, Soerjomataram I, et al., Global cancer statistics 2018: GLOBOCAN estimates of incidence and mortality worldwide for 36 cancers in 185 countries, CA Cancer J Clin 2018; 68: 394-424.

[2] Herbst RS, Heymach JV, and Lippman SM, Lung cancer, N Engl J Med 2008; 359: 1367-1380.

[3] Lynch TJ, Bell DW, Sordella R, et al., Activating mutations in the epidermal growth factor receptor underlying responsiveness of non-small-cell lung cancer to gefitinib, N Engl J Med 2004; 350: 2129-2139.

[4] Pao W, and Chmielecki J, Rational, biologically based treatment of EGFR-mutant non-small-cell lung cancer, Nat Rev Cancer 2010; 10: 760-774.

[5] Ettinger DS, Wood DE, Aisner DL, et al., Non-Small Cell Lung Cancer, Version 5.2017, NCCN Clinical Practice Guidelines in Oncology, J Natl Compr Canc Netw 2017; 15: 504-535.

[6] Pao W, Miller VA, Politi KA, et al., Acquired resistance of lung adenocarcinomas to gefitinib or erlotinib is associated with a second mutation in the EGFR kinase domain, PLoS Med 2005; 2: e73.

[7] Pao W, Wang TY, Riely GJ, et al., KRAS mutations and primary resistance of lung adenocarcinomas to gefitinib or erlotinib, PLoS Med 2005; 2: e17.

[8] Engelman JA, Mukohara T, Zejnullahu K, et al., Allelic dilution obscures detection of a biologically significant resistance mutation in EGFR-amplified lung cancer, J Clin Invest 2006; 116: 2695-2706.

[9] Engelman JA, Zejnullahu K, Mitsudomi T, et al., MET amplification leads to gefitinib resistance in lung cancer by activating ERBB3 signaling, Science 2007; 316: 1039-1043.

[10] Sequist LV, Waltman BA, Dias-Santagata D, et al., Genotypic and histological evolution of lung cancers acquiring resistance to EGFR inhibitors, Sci Transl Med 2011; 3: 75ra26.

[11] Ornitz DM, and Itoh N, The Fibroblast Growth Factor signaling pathway, Wiley Interdiscip Rev Dev Biol 2015; 4: 215-266. 
[12] Beenken A, and Mohammadi M, The FGF family: biology, pathophysiology and therapy, Nat Rev Drug Discov 2009; 8: 235-253.

[13] Wang K, Ji W, Yu Y, et al., FGFR1-ERK1/2-SOX2 axis promotes cell proliferation, epithelialmesenchymal transition, and metastasis in FGFR1-amplified lung cancer, Oncogene 2018; 37: 5340-5354.

[14] Konermann S, Brigham MD, Trevino AE, et al., Genome-scale transcriptional activation by an engineered CRISPR-Cas9 complex, Nature 2015; 517: 583-588.

[15] Vad-Nielsen J, Lin L, Bolund L, et al., Golden Gate Assembly of CRISPR gRNA expression array for simultaneously targeting multiple genes, Cell Mol Life Sci 2016; 73: 4315-4325.

[16] Weiss J, Sos ML, Seidel D, et al., Frequent and focal FGFR1 amplification associates with therapeutically tractable FGFR1 dependency in squamous cell lung cancer, Sci Transl Med 2010; 2: 62 ra93.

[17] Malchers F, Dietlein F, Schottle J, et al., Cell-autonomous and non-cell-autonomous mechanisms of transformation by amplified FGFR1 in lung cancer, Cancer Discov 2014; 4: 246-257.

[18] Facchiano A, Russo K, Facchiano AM, et al., Identification of a novel domain of fibroblast growth factor 2 controlling its angiogenic properties, J Biol Chem 2003; 278: 8751-8760.

[19] Turner N, Pearson A, Sharpe R, et al., FGFR1 amplification drives endocrine therapy resistance and is a therapeutic target in breast cancer, Cancer Res 2010; 70: 2085-2094.

[20] Matsumoto K, Arao T, Hamaguchi T, et al., FGFR2 gene amplification and clinicopathological features in gastric cancer, $\mathrm{Br} \mathrm{J}$ Cancer 2012; 106: 727-732.

[21] Lu J, Zhong H, Chu T, et al., Role of anlotinib-induced CCL2 decrease in anti-angiogenesis and response prediction for nonsmall cell lung cancer therapy, Eur Respir J 2019; 53:

[22] Lin B, Song X, Yang D, et al., Anlotinib inhibits angiogenesis via suppressing the activation of VEGFR2, PDGFRbeta and FGFR1, Gene 2018; 654: 77-86.

[23] Ashton S, Song YH, Nolan J, et al., Aurora kinase inhibitor nanoparticles target tumors with favorable therapeutic index in vivo, Sci Transl Med 2016; 8: 325ra317.

[24] Wang C, Chen J, Cao W, et al., Aurora-B and HDAC synergistically regulate survival and proliferation of lymphoma cell via AKT, mTOR and Notch pathways, Eur J Pharmacol 2016; 779: 1-7.

[25] Ambrogio C, Gomez-Lopez G, Falcone M, et al., Combined inhibition of DDR1 and Notch signaling is a therapeutic strategy for KRAS-driven lung adenocarcinoma, Nat Med 2016; 22: 270-277.

[26] Kakiuchi-Kiyota S, Lappin PB, Heintz C, et al., Expression of proto-oncogene cFMS protein in lung, breast, and ovarian cancers, Appl Immunohistochem Mol Morphol 2014; 22: 188-199. 
[27] Koizumi F, Shimoyama T, Taguchi F, et al., Establishment of a human non-small cell lung cancer cell line resistant to gefitinib, Int J Cancer 2005; 116: 36-44.

[28] Zhang N, Zeng Y, Du W, et al., The EGFR pathway is involved in the regulation of PD-L1 expression via the IL-6/JAK/STAT3 signaling pathway in EGFR-mutated non-small cell lung cancer, Int J Oncol 2016; 49: $1360-1368$.

[29] Paez JG, Janne PA, Lee JC, et al., EGFR mutations in lung cancer: correlation with clinical response to gefitinib therapy, Science 2004; 304: 1497-1500.

[30] Mok TS, Wu YL, Thongprasert S, et al., Gefitinib or carboplatin-paclitaxel in pulmonary adenocarcinoma, N Engl J Med 2009; 361: 947-957.

[31] Turner N, and Grose R, Fibroblast growth factor signalling: from development to cancer, Nat Rev Cancer 2010; 10: 116-129.

[32] Knights V, and Cook SJ, De-regulated FGF receptors as therapeutic targets in cancer, Pharmacol Ther 2010; 125: 105-117.

[33] Wesche J, Haglund K, and Haugsten EM, Fibroblast growth factors and their receptors in cancer, Biochem J 2011; 437: 199-213.

\section{Figures}


$\mathbf{A}$

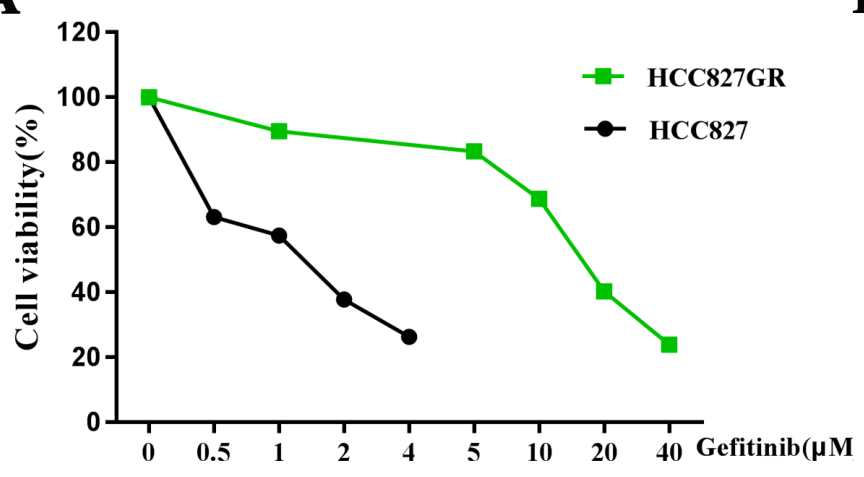

B
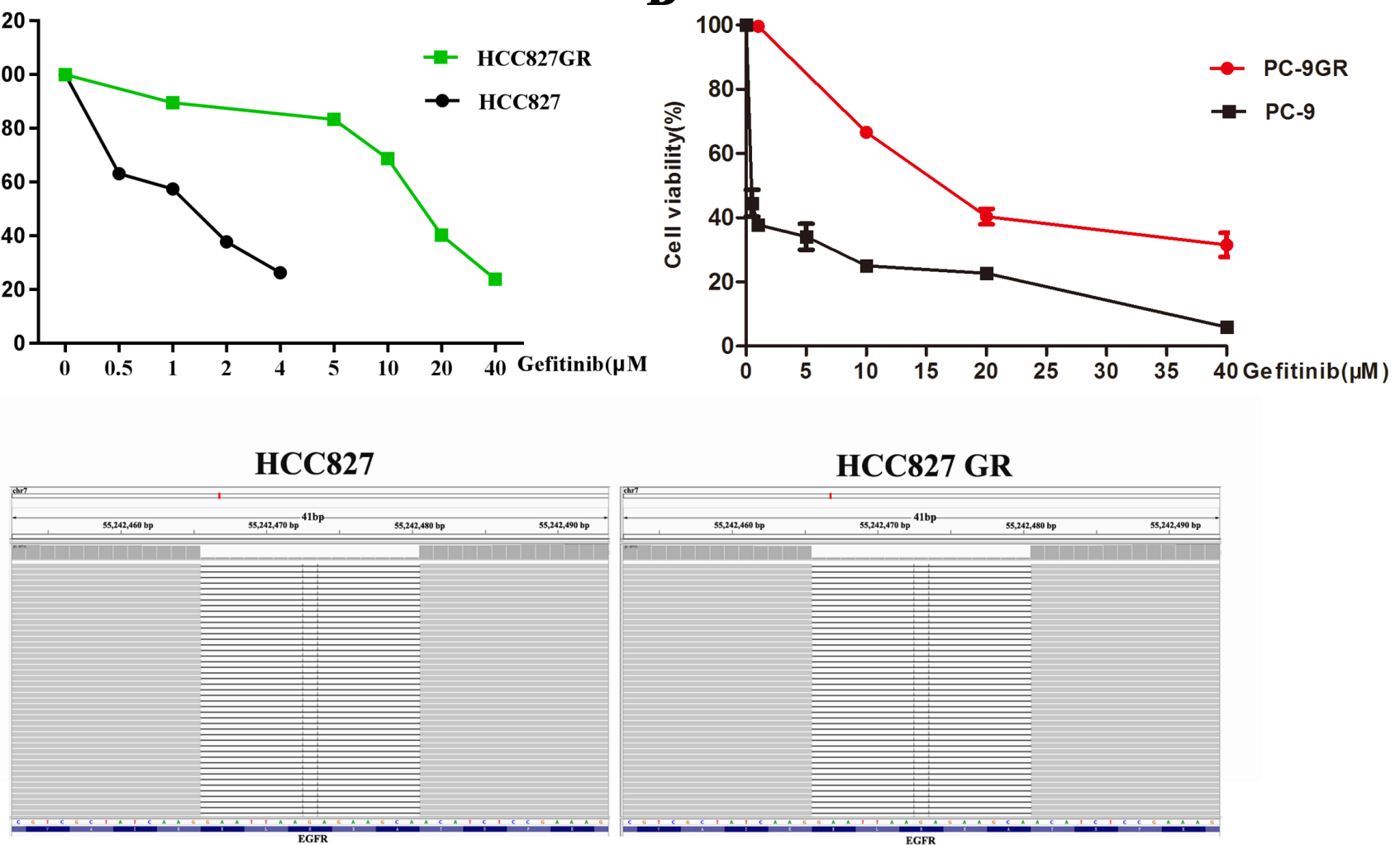

p.746_750del (c.2236_2250del GAATTAAGAGAAGCA

PC-9

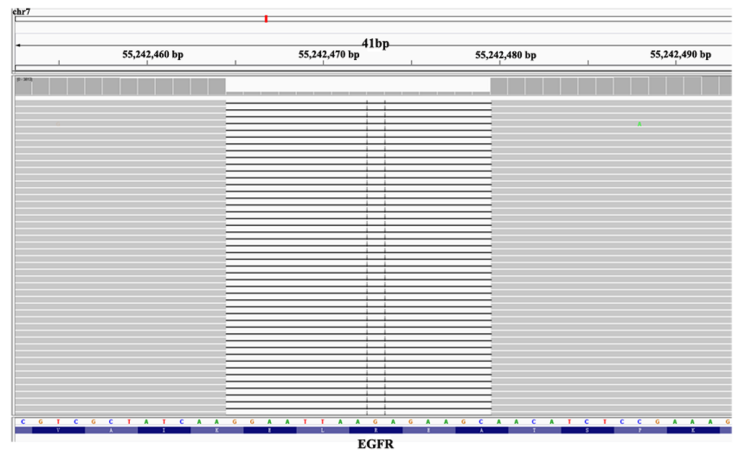

p.745_750del (c.2235_2249del GGAATTAAGAGAAGC
HCC827 GR

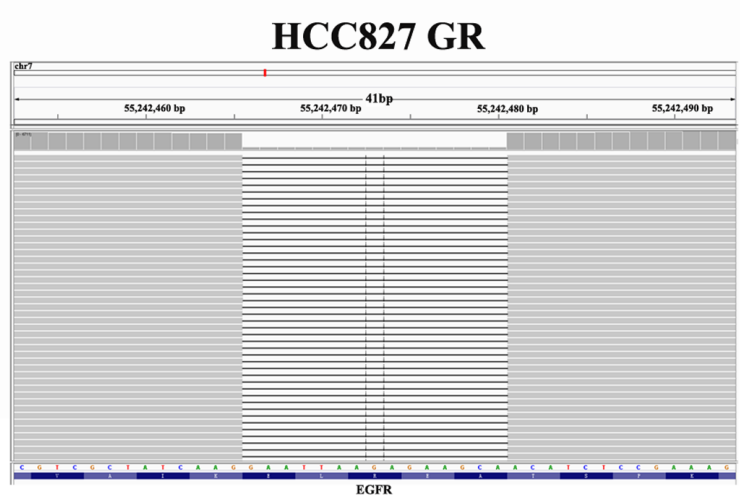

p.745_750del (c.2235_2249del GGAATTAAGAGAAGC

PC-9 GR

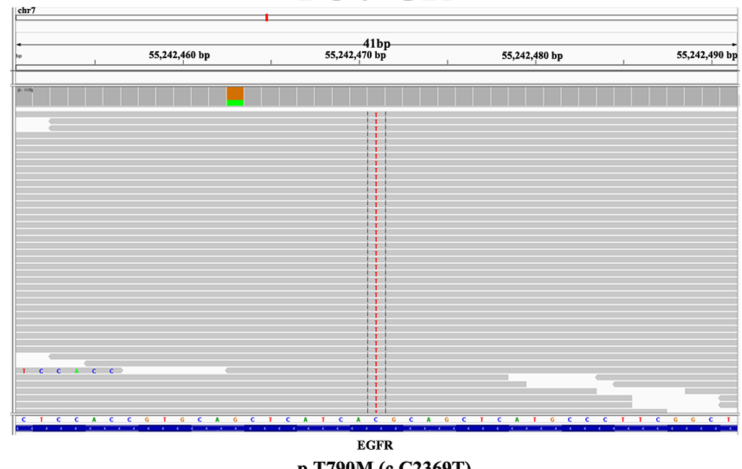

\section{Figure 2}

Gefitinib resistance in HCC827 GR cells is not due to EGFR T790M mutation. (A) and (B) HCC827, PC-9, HCC827 GR and PC-9 GR cells were treated with gefitinib at different concentrations, and CCK-8 assays were used to assess cell viability. (C) Next-generation DNA sequencing was used to analyse EGFR for the DNA substitution corresponding to the T790M mutation. 
$\mathbf{A}$
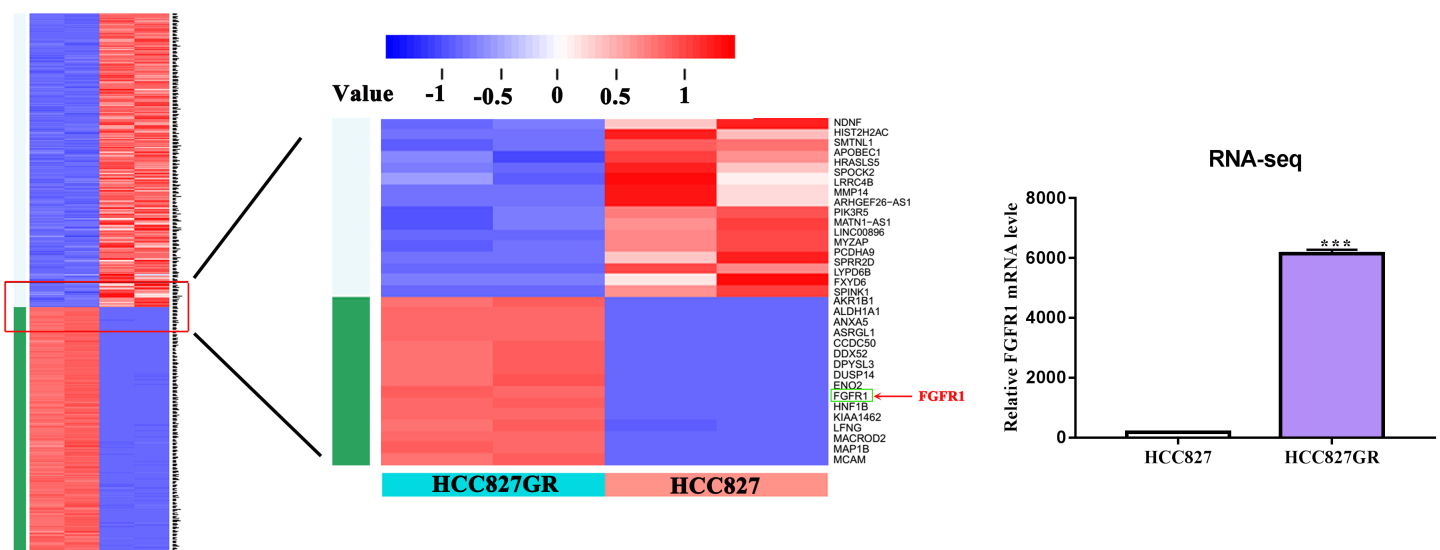

B
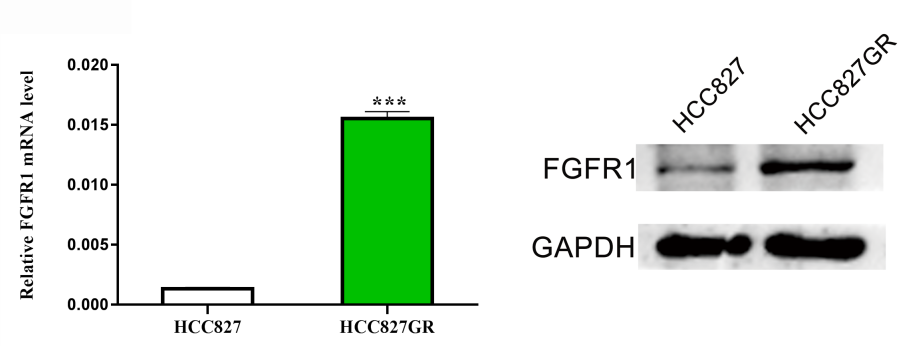

C
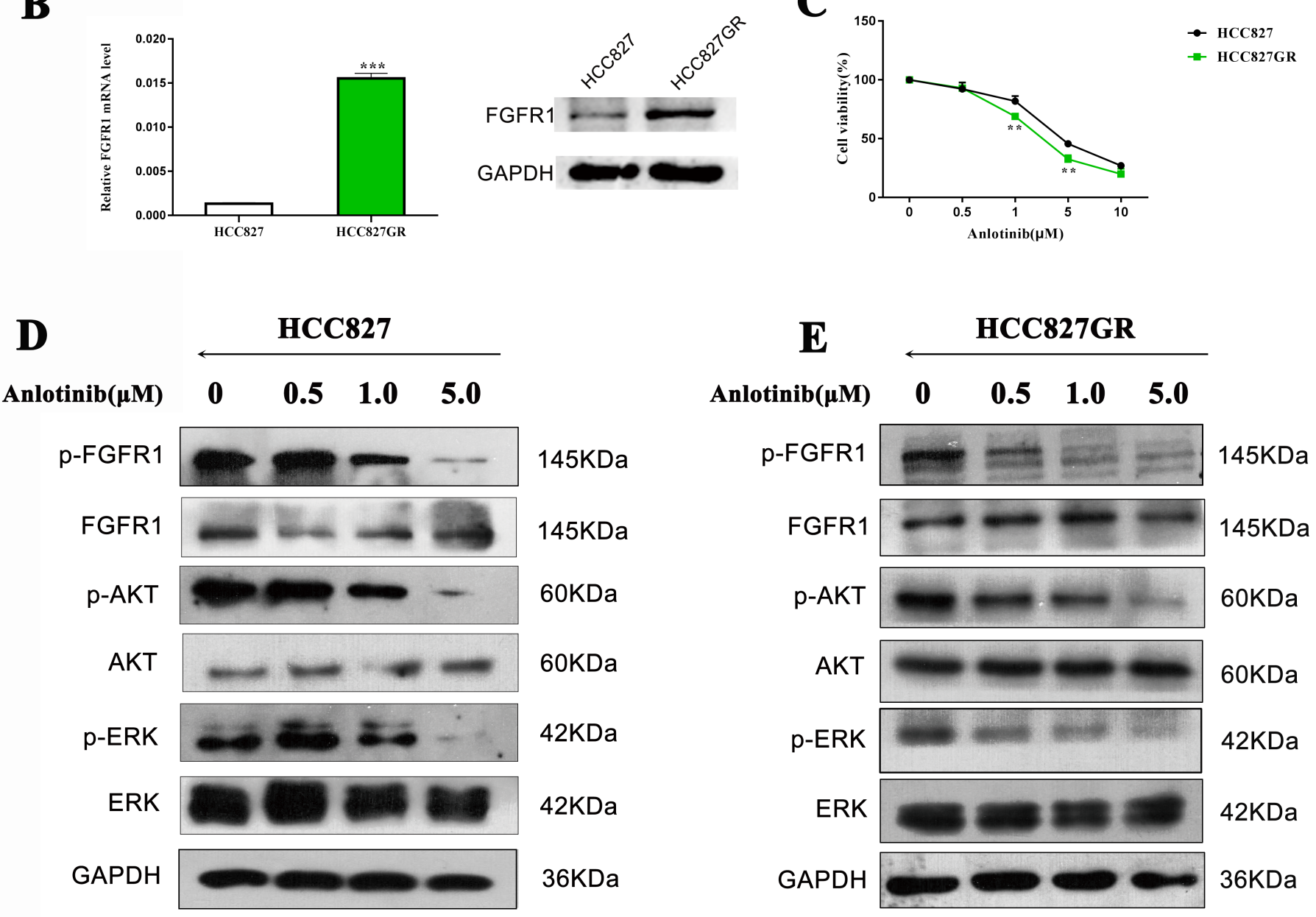

Figure 3

FGFR1 expression is higher in HCC827 GR cells than in parental HCC827 cells. (A) Microarray analysis was used to analyse differences in mRNA expression levels between HCC827 and HCC827 GR cells. (B) The expression level of FGFR1 was determined by quantitative real-time PCR and Western blot analysis. (C) CCK-8 assays were performed to investigate the effects of anlotinib on HCC827 and HCC827 GR cells 
in vitro. (D) and (E) Western blot analysis was used to detect the effect of anlotinib on p-FGFR1, p-AKT and p-ERK in HCC827 and HCC827 GR cells. (** $\mathrm{P}<0.01)$.

$\mathbf{A}$
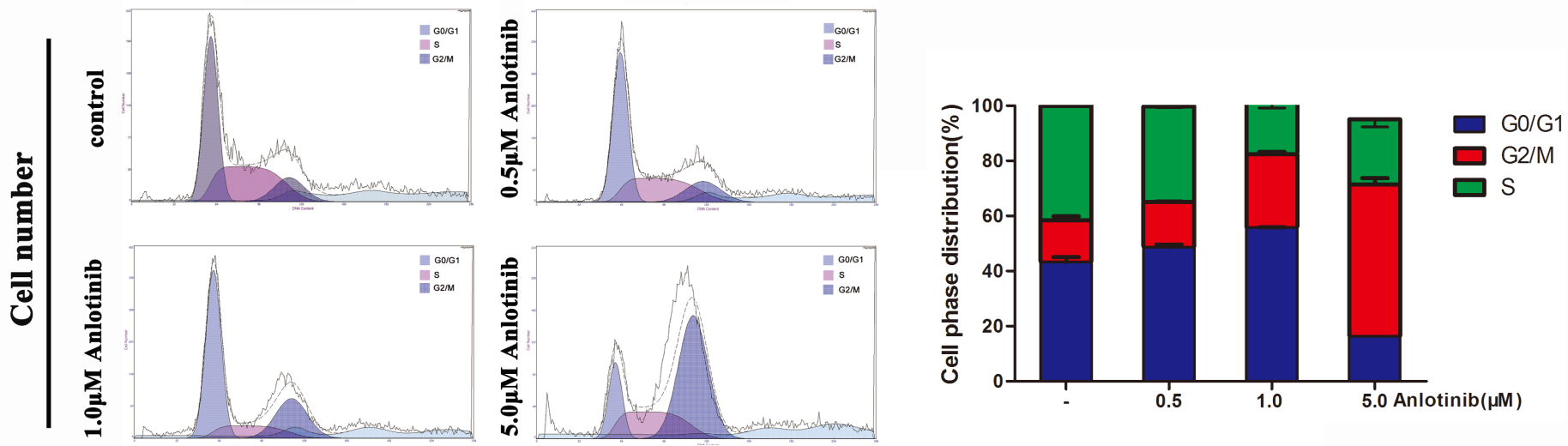

B

DNA content
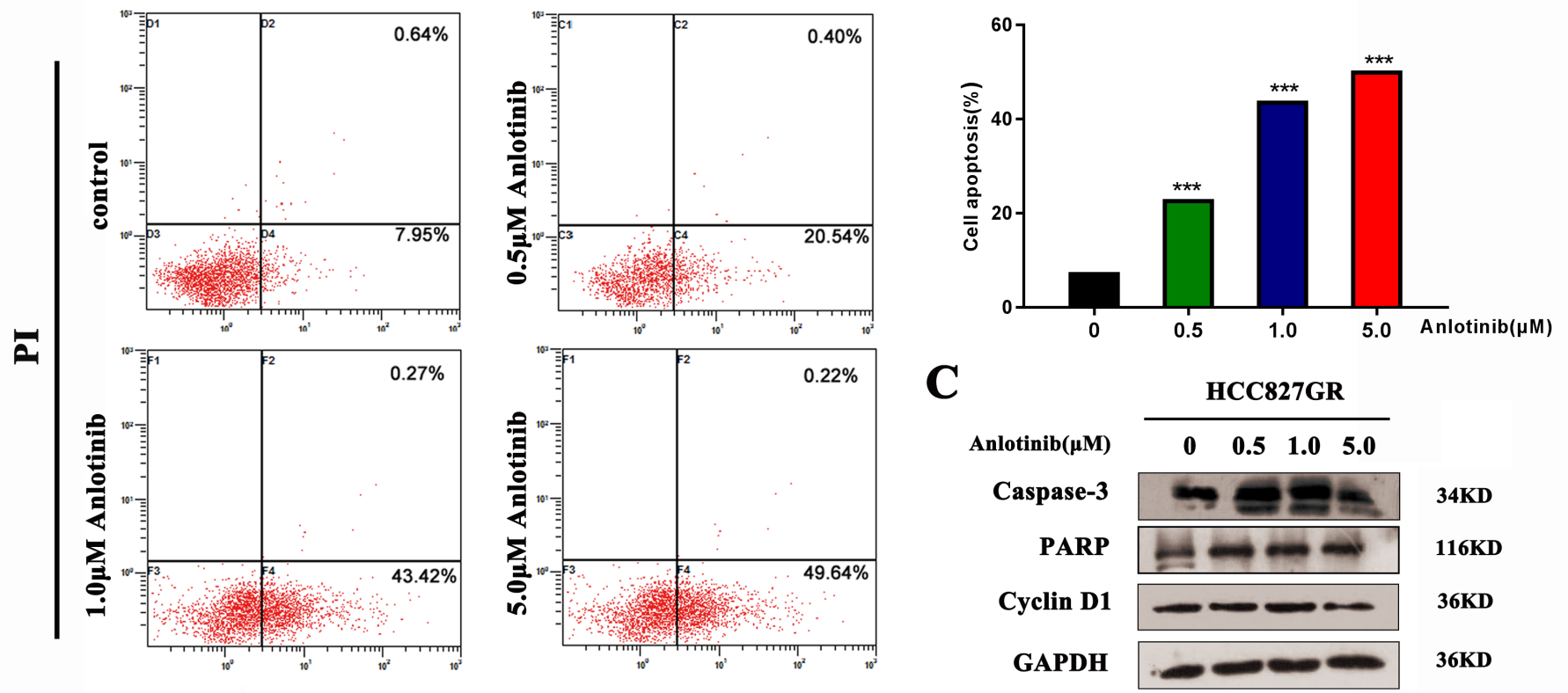

C

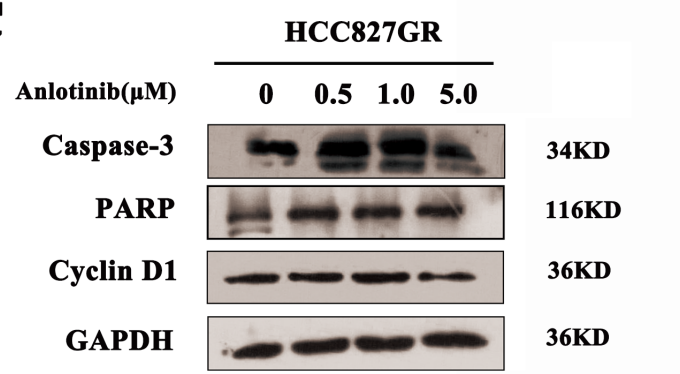

Figure 5

Anlotinib induces apoptosis and cycle arrest in HCC827 GR cells. (A) and (B) Fluorescence-activated cell sorting (FACS) analysis was performed to investigate the effects of anlotinib on the cell cycle and apoptosis in HCC827 GR cells. (C) Western blot analysis was used to detect the effect of anlotinib on Caspase-3, PARP and Cyclin D1 expression in HCC827 GR cells. $(* \star * P<0.001)$. 
A

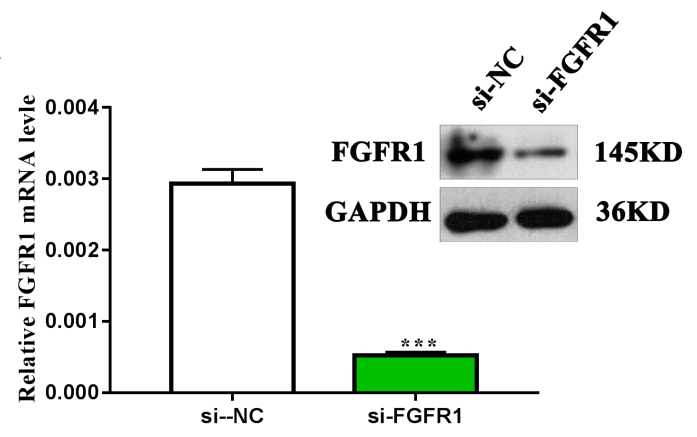

C

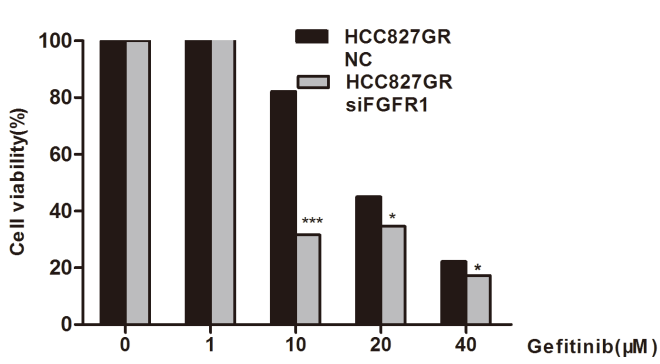

$\mathbf{E}$

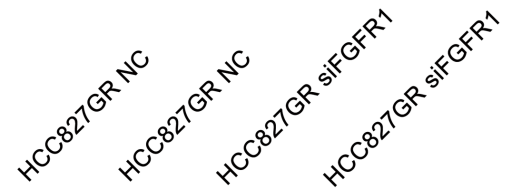

Gefitinib(5 $\mu M)$

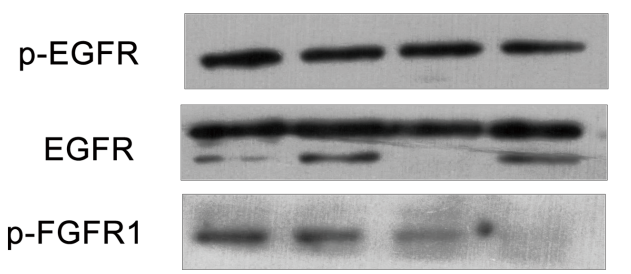

FGFR

p-AKT

AKT

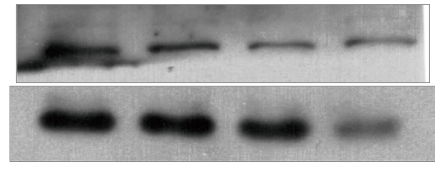

p-ERK
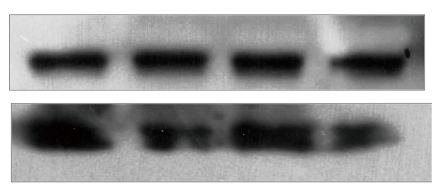

ERK

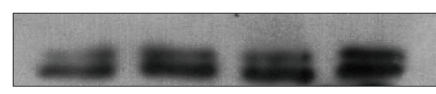

GAPDH

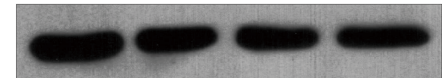

170KDa

170KDa

145KDa

60KDa

60KDa

42KDa

42KDa

36KDa

F

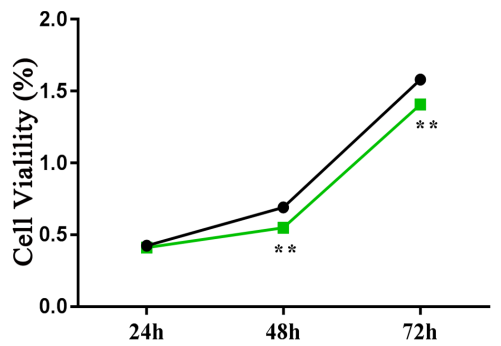

$\rightarrow$ si- NC

- si-FGFR 1

D
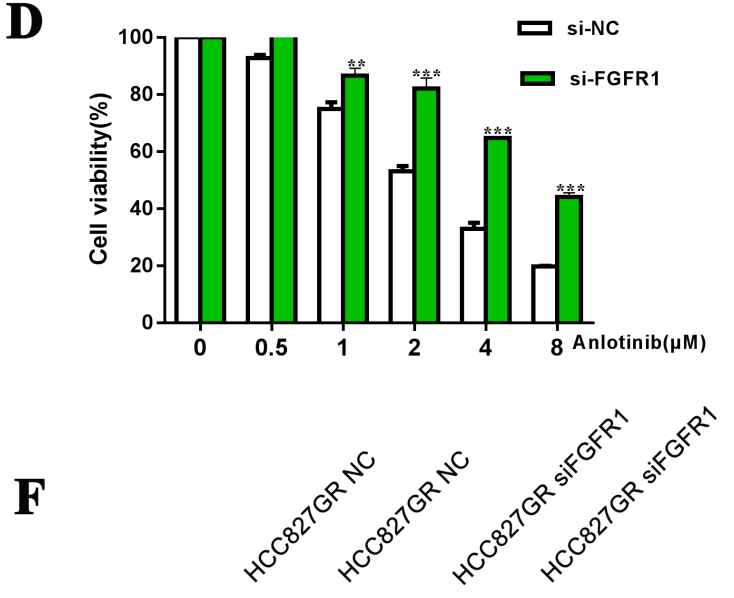

Gefitinib(10 $\mu M)$

p-EGFR

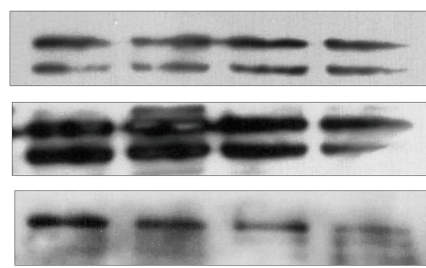

170KDa

EGFR

170KDa

p-FGFR1

145KDa

145KDa FGFR1

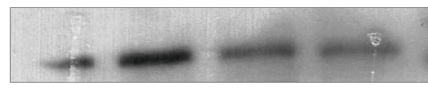

$145 \mathrm{KDa}$

p-AKT

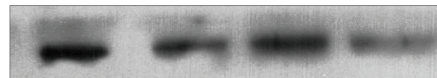

60KDa

AKT

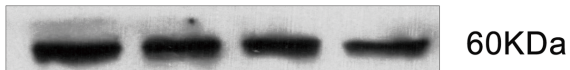

p-ERK

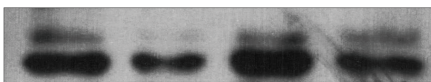

42KDa

ERK

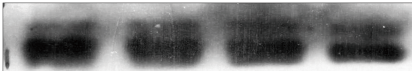

42KDa

GAPDH

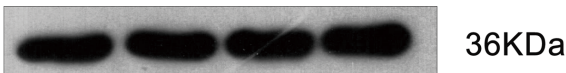

\section{Figure 8}

Knockdown of FGFR1 reverses gefitinib resistance in HCC827 GR cells. (A) qRT-PCR and Western blot analysis were used to detect the expression of FGFR1 in HCC827 GR cell lines after transfection with siFGFR1. (B) The cell viability of HCC827 GR cells transfected with si-FGFR1 or si-NC was detected by CCK8 assays. (C) The cell viability of HCC827 GR cells transfected with si-FGFR1 or si-NC treated with gefitinib or anlotinib was detected by CCK-8 assays. (E) and (F) Western blot analysis was used to detect 
the effect of anlotinib on the gefitinib-induced downregulation of p-EGFR, p-AKT and p-ERK in HCC827 GR cells transfected with si-FGFR1 or si-NC.

$\mathbf{A}$

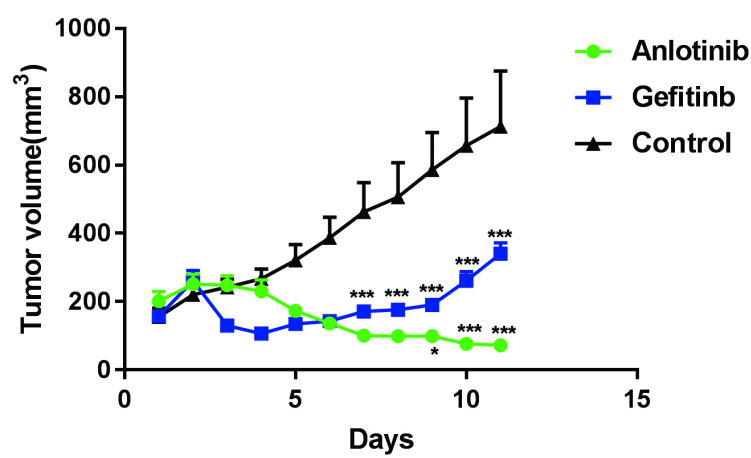

C

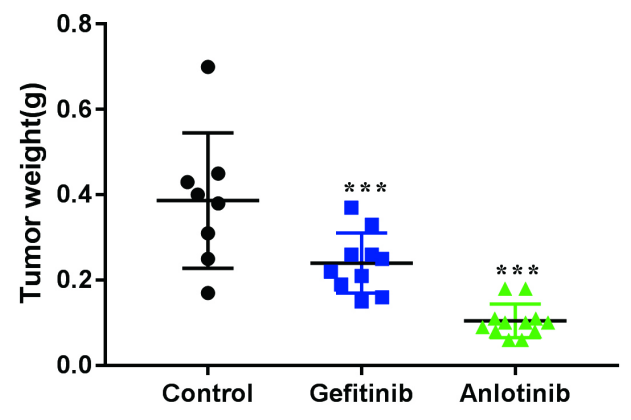

B

\section{Control}

Gefitinib

Anlotinib

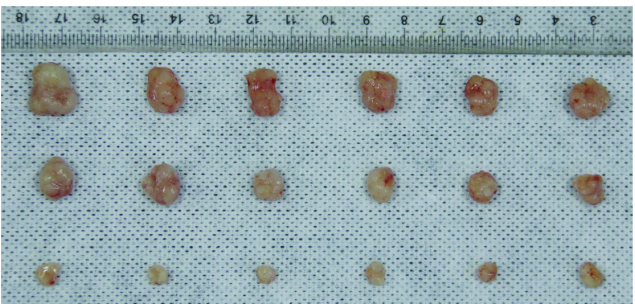

D

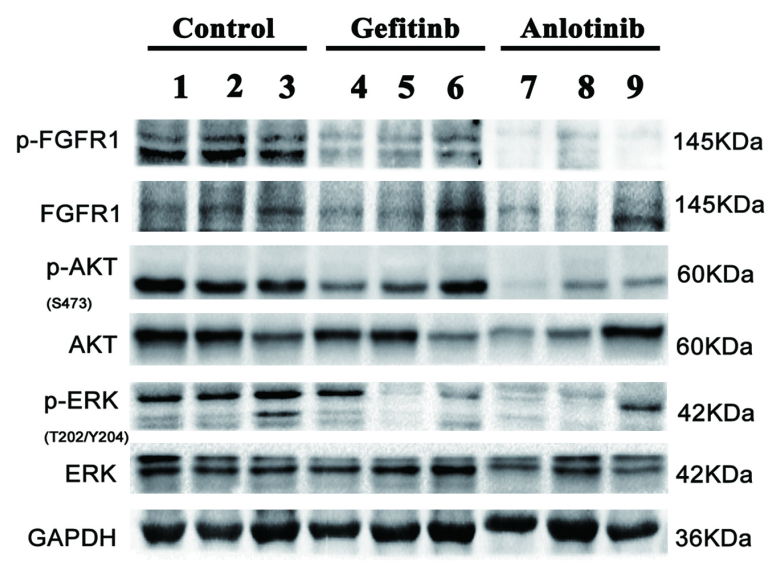

Figure 10

Anlotinib inhibits the growth of HCC827 GR cells in vivo. (A) The tumour volumes were measured at the indicated time intervals and calculated. See the Materials and methods section for details. (B) and (C) At the end of treatment, the tumours were excised, photographed as indicated and weighed. (D) Western blotting was used to analyse the levels of p-FGFR, p-AKT and p-ERK in tumour tissues from different treatment groups. (*** $\mathrm{P}<0.001)$.

\section{Supplementary Files}

This is a list of supplementary files associated with this preprint. Click to download.

- SupplementalTable1.docx

- SupplementalTable1.docx

- SupplementalFigure1.tif

- SupplementalFigure1.tif 